\title{
Coupled channels approach to photo-meson production on the nucleon
}

\author{
Horst Lenske ${ }^{1, \star}$ \\ ${ }^{1}$ Institut für Theoretische Physik, Justus-Liebig-Universität Gießen
}

\begin{abstract}
.
The coupled channels Lagrangian approach of the Giessen model (GiM) for meson production on the nucleon is discussed and applied to a selected set of meson production channels on the nucleon, ranging from $\pi N \rightarrow \pi N$ and $\gamma N \rightarrow \pi N$, eta-production and associated strangeness production to $2 \pi N$ channels in the resonance energy region. We present an updated coupled-channel analysis of eta-meson production including all recent photoproduction data on the proton. The dip structure observed in the differential cross sections at c.m. energies $\mathrm{W}=1.68 \mathrm{GeV}$ is explained by destructive interference between the $S_{11}(1535)$ and $S_{11}(1560)$ states, not confirming the postulated sharp state. Kaon production on the nucleon is investigated in $K \Lambda$ and $K \Sigma$ exit channels. The approach to $2 \pi N$ production has been significantly improved by using the isobar approximation with $\sigma N$ and $\pi \Delta_{1232}$ intermediate states. Three-body unitarity is maintained up to interference between the isobar subchannels. We obtain $R_{\sigma N}(1440)=27_{-9}^{+4} \%$ and $R_{\pi \Delta}(1440)=12_{-3}^{+5} \%$ for the $\sigma N$ and $\pi \Delta_{1232}$ decay branching ratios of $N^{*}(1440)$ respectively. The extracted $\pi N$ inelasticities and reaction amplitudes are consistent with the results of other groups.
\end{abstract}

\section{Introduction}

The discovery of nucleon resonances in the early pionnucleon scattering experiments provided first indications for a complicated intrinsic structure of the nucleon. With establishing the quark picture of hadrons and developments of the constituent quark models the interest in the study of the nucleon excitation spectra was renewed. Soon after, it was realized that there was an obvious discrepancy between the number of resonances predicted by theory and those identified experimentally. Since then, the problem of missing resonances is a major issue of baryon spectroscopy. Final answers about the number of excited states of the nucleon and their properties are still pending. Solutions are searched for both experimentally and theoretically. On the theory side constituent quark (CQM) models, lattice QCD and Dyson-Schwinger approaches have been developed to describe and predict the nucleon resonance spectra. The main problem remains, however, a serious disagreement between the theoretical calculations and the experimentally observed baryon spectra. This concerns both the number and the properties of excited states.

The investigation of properties of nucleon resonances remains one of the primary goals of modern hadron physics. The main information about the hadron spectra comes from the analysis of scattering data. Coupledchannel approaches have proven to be an efficient tool to extract baryon properties from experiment. The Giessen coupled-channel model (GiM) has been developed for a combined analysis of pion- and photon-induced reactions on the nucleon, $(\pi / \gamma)+N$, for extracting properties of nucleon resonances. The applications range from investigations of the elastic and inelastic $\pi N$ and $\pi N^{*}$ channels [1] to $\omega N[2], \eta N[3,4]$ production as well as the strangeness channels $K \Lambda$ [5] and $K \Sigma$ [6]. The $2 \pi N$ channels were investigated recently in [7]. Here we review central issues of the Giessen approach and present results for selected reactions. In section 2 we introduce the underlying field theoretical model, being based on a phenomenological Lagrangian density for baryons, mesons, and the photon and their interactions. General theoretical aspects of high-spin matter fields are discussed in section 3 with special emphasis on the gauge properties of $s=\frac{3}{2}$ and $s=\frac{5}{2}$. Applications and results of our approach on the production of kaon- and eta-mesons are discussed in section 4 and section 5, respectively. Our latest investigations on doublepion production channels are discussed in section 6 . In section 7 we summarize the achievements of the Giessen model. As an illustrative overview we present already here in Fig. 1 our results on the total cross sections in the various hadronic reaction channels.

\section{The Giessen coupled channels model for baryon spectroscopy}

Here we briefly outline the main ingredients of the model. More details can be found in [1,3-7]. We need to solve the Bethe-Salpeter (BS) equation for the scattering amplitude:

$$
\begin{aligned}
& M\left(p^{\prime}, p ; w\right)=V\left(p^{\prime}, p ; w\right) \\
& +\int \frac{\mathrm{d}^{4} q}{(2 \pi)^{4}} V\left(p^{\prime}, q ; w\right) G_{B S}(q ; w) M(q, p ; w),
\end{aligned}
$$




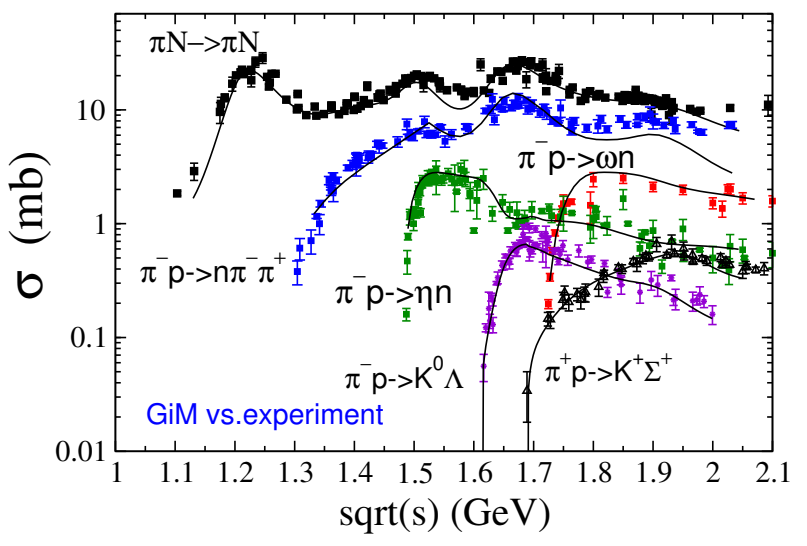

Figure 1. Total cross sections for pion-induced reactions. Results of the Giessen model are compared to experimental data.

where $w=\sqrt{s}$ is the available center-of-mass energy. Here, $p(k)$ and $p^{\prime}\left(k^{\prime}\right)$ are the incoming and outgoing baryon (meson) four-momenta. After splitting the two-particle BS propagator $G_{B S}$ into its real and imaginary parts, we introduce the $K$-matrix given schematically by $K=V+\int V \operatorname{Re} G_{B S} M$. Then $M$ is given by $M=K+i \int M \operatorname{Im} G_{B S} K$. Since the imaginary part of $G_{B S}$ is given by the on-shell part, the reaction matrix $T$, defined via the scattering matrix $S=1+2 i T$, can now be calculated from $K$ after a partial wave decomposition $(P W D)$ into total spin $J$, parity $P$, and isospin $I$ via matrix inversion:

$$
T\left(p^{\prime}, p ; w\right)=\left(1-i \rho(w) K\left(p^{\prime}, p ; w\right)\right)^{-1} K\left(p^{\prime}, p ; w\right),
$$

where $\rho(w)$ is an appropriately chosen phase space factor. Hence unitarity is fulfilled as long as $K$ is Hermitian. For simplicity we apply the so-called $K$-matrix Born approximation, which means that we neglect the real part of $G_{B S}$ and thus $K$ reduces to $K=V$. The validity of this approximation was tested a long time ago by Pearce and Jennings [8].

The potential $V$ is built up by a sum of $s$-, $u$-, and $t$-channel Feynman diagrams by means of effective Lagrangians which are not shown here but can be found in very detail in [9] and the previously cited references. Certain aspects of the more involved cases of spin- $\frac{3}{2}$ and spin$\frac{5}{2}$ resonances are discussed below in section 3 . In all reaction channels the non-resonant background contributions to the scattering amplitudes are consistently derived from the $u$ - and $t$-channel diagrams, thus reducing the number of free parameters greatly. In addition, each vertex is multiplied by a cutoff form factor:

$$
F\left(q^{2}\right)=\frac{\Lambda_{q}^{4}}{\Lambda_{q}^{4}+\left(q^{2}-m_{q}^{2}\right)^{2}},
$$

where $m_{q}\left(q^{2}\right)$ denotes the mass (four-momentum squared) of the off-shell particle. To reduce the number of parameters the cutoff value $\Lambda_{q}$ is chosen to be identical for all final states. We only distinguish between the nucleon cutoff $\left(\Lambda_{N}\right)$, the resonance cutoffs $\left(\Lambda_{s}\right)$, where a common cutoff is used for all baryons within the same spin-multiplet $s \in\left\{\frac{1}{2}, \frac{3}{2}, \frac{5}{2} \ldots\right\}$, and the $t$-channel cutoff $\left(\Lambda_{t}\right)$.
A considerable numerical simplification is obtained by the afore mentioned $K$-matrix approximation. Using the partial wave decomposition the integral over $d \Omega_{q}$ can be calculated analytically. Then Eq. (1) reduces to a linear system of coupled equations for the partial wave scattering amplitudes:

$$
\begin{aligned}
T_{f i}^{J P}(w) & =K_{f i}^{J P}(w) \\
& +\mathrm{i} \sum_{j} \int_{\mu_{j 0}^{2}}^{\infty} d \mu_{j}^{2} A_{j}\left(\mu_{j}\right) T_{f j}^{J P}\left(\mu_{j}\right) K_{j i}^{J P}\left(\mu_{j}\right)(4)
\end{aligned}
$$

where $K=V$ and $f, i, j$ denote the final, initial, and intermediate meson - baryon channels, respectively. The photo-production reaction channels are treated perturbatively in leading order of the $\gamma N$ and $\gamma N^{*}$ vertices, respectively. The spectral functions in the meson-meson $(\pi, K, \sigma, \rho \ldots)$ and the meson-baryon isobar channels, as e.g the $\Delta$ resonance, are denoted by $A_{j}\left(\mu_{j}^{2}\right)$, being integrated over the energy $\mu_{j}$ available in the isobar subsystem starting at the isobar threshold energy $\mu_{j 0}$. For stable particles the spectral functions reduce to trivial delta-functions, projecting the integrand on the particles's mass. Since the two-pion discontinuities are taken into account the threebody unitarity in the form of the optical theorem if fulfilled up to interference terms between different isobar subchannels [7].

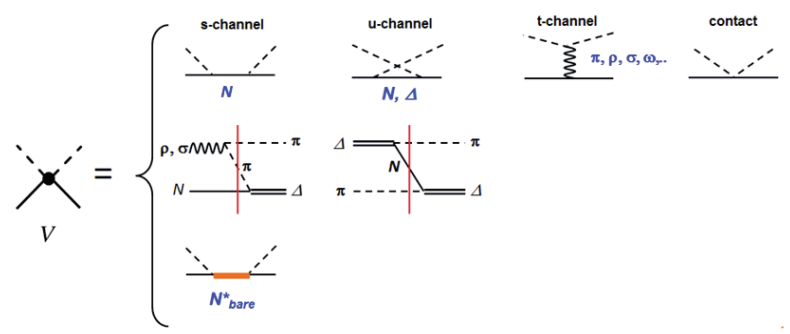

Figure 2. The structure of the tree-level interaction potential $V . s-, u-$, and $t-$ channel interactions defining the non-resonant background contributions are shown in the first line, including contact terms which are chosen such that gauge invariance is assured. The so-called $z$-diagrams, displayed in the second line, are generic for the double-pion channels. $s$ - channel resonance interactions are depicted in the last line. Time is running from left to right.

\section{Theory of high-spin fermionic fields}

\subsubsection{Gauge properties of spin-3/2 fields}

It is well known that the wave equation for the free spin$\frac{3}{2}$ field [10] being written in a general form depends on one free parameter $A$ (see e.g. [15]). The commonly used Rarita-Schwinger theory [10] corresponds to the special choice $A=-1$. While the so-called Pascalutsa-coupling removes the unwanted degrees of freedom from the RaritaSchwinger propagator it leaves the problem unsolved in the more general case $A \neq-1$ resulting in the appearance of 'off-shell' components, for example in the $\pi N$ scattering amplitude. Hence, further investigations of the general 
properties of the interacting spin- $\frac{3}{2}$ fields are of great importance. We have discussed the origin of this problem and shown how it to solved it. Two alternative approaches were indicated:

- the first solution consists in constructing a coupling which includes higher derivatives of the spin- $\frac{3}{2}$ field.

- alternatively, advantage may be taken on the generalization of the original gauge invariant interaction to arbitrary values of $A \neq-1$.

In the latter case the obtained Lagrangian depends on one free parameter which also appears in the free field formalism. However, the physical observables should not depend on this parameter. Hence, the matrix element corresponding to the $\pi N$ scattering at tree level does not contain an off-shell background. Rarita and Schwinger suggested a set of constraints which the free spin- $\frac{3}{2}$ field should obey [10]

$$
\begin{aligned}
& \gamma^{v} \psi_{v}(x)=0, \\
& \partial^{v} \psi_{v}(x)=0,
\end{aligned}
$$

provided that also the Dirac equation $(\not p-m) \psi_{v}(p)=0$ is fulfilled. In a consistent theory the set of equations Eq. (5) should follow from the equation of motion obtained from the corresponding Lagrangian. The Lagrangian of the free spin- $\frac{3}{2}$ field can be written in a general form as follows (see, e.g., [15] and references therein)

$$
\mathcal{L}_{0}^{\frac{3}{2}}=\bar{\Delta}_{\mu}(x) \Lambda^{\mu v} \Delta_{v}(x),
$$

where $\Delta_{v}(x)$ stands for the spin- $\frac{3}{2}$ field and the $\Lambda^{\mu \nu}$ operator is

$$
\begin{aligned}
\Lambda^{\mu \nu}=\quad & (\mathrm{i} \not \partial-m) g^{\mu \nu}+\mathrm{i} A\left(\gamma^{\mu} \partial^{v}+\gamma^{\nu} \partial^{\mu}\right) \\
& +\frac{\mathrm{i}}{2}\left(3 A^{2}+2 A+1\right) \gamma^{\mu} \not \partial \gamma^{\nu} \\
& +m\left(3 A^{2}+3 A+1\right) \gamma^{\mu} \gamma^{\nu},
\end{aligned}
$$

where $A$ is the afore mentioned arbitrary free parameter, subject only to the restriction $A \neq-\frac{1}{2}$. The propagator of the free spin- $\frac{3}{2}$ field can be obtained as a solution of the equation, e.g. in momentum space,

$$
\Lambda_{\mu \rho}(p) g^{\rho \sigma} G_{\sigma v}(p)=g_{\mu \nu}
$$

The propagator $G_{\sigma v}(p)$ can be written as an expansion in terms of the spin projection operators $\mathcal{P}_{\mu \nu}^{\frac{3}{2}}(p), \mathcal{P}_{11 ; \mu \nu}^{\frac{1}{2}}$, $\mathcal{P}_{22 ; \mu \nu}^{\frac{1}{2}}, \mathcal{P}_{12 ; \mu \nu}^{\frac{1}{2}}(p)$, and $\mathcal{P}_{21 ; \mu \nu}^{\frac{1}{2}}(p)$ [11]. The first three operators correspond to different irreducible representations of spin-vector whereas the last two account for a mixing between two spin- $\frac{1}{2}$ representations.

Without going too deep into the mathematical details, we constrain the discussion here to the consequences for interaction vertices. A commonly used $\Delta N \pi$-coupling is $\mathcal{L}_{\text {int }} \sim \bar{\psi}_{N} \theta(z)^{\nu \mu} \Delta_{\mu} \partial_{v} \pi$ with $\theta^{\mu \nu}(z)=g^{\mu \nu}+z \gamma^{\mu} \gamma^{\nu}$. The free parameter $z$ is used to control the off-shell contributions to the interaction vertex but does not affect the pole term. In order to remove the dependence on $z$ (or, likewise, $A$ ) we eliminate the unwanted degrees of freedom by using a gauge-invariant coupling to the spin- $\frac{3}{2}$ field as explained in [11]. The modified $\Delta N \pi$ interaction Lagrangian can be written as follows ${ }^{1}$

$$
\begin{array}{r}
\mathcal{L}_{P}=\frac{\mathrm{g}_{\Delta N \pi}}{m_{\pi} m_{N}} \bar{\psi}_{N}(x) \gamma_{5} \gamma_{\mu} T_{\Delta}^{\mu v}(x) \partial_{v} \pi(x)+\text { h.c. }, \\
T_{\Delta}^{\mu v}(x)=\frac{1}{2} \epsilon^{\mu v \rho \sigma}\left(\partial_{\rho} \Delta_{\sigma}(x)-\partial_{\sigma} \Delta_{\rho}(x)\right),
\end{array}
$$

where $\epsilon^{\mu \nu \rho \sigma}$ is the fully antisymmetric Levi-Civita tensor. The tensor $T_{\mu \nu}(x)$ is invariant under the gaugetransformations $\Delta_{v}(x) \rightarrow \Delta_{v}(x)+\partial_{\nu} \xi(x)$ where $\xi(x)$ is an arbitrary spinor field. Hence, $T_{\mu \nu}(x)$ behaves like a conserved current with the constraint $\partial_{\mu} T_{\Delta}^{\mu v}(x)=0$. The coupling defined in Eq. (9) guarantees that the so-called offshell background does not contribute to the physical observables provided that the free spin- $\frac{3}{2}$ propagator is chosen in the special form corresponding to $A=-1$. This, however, does not hold in the general case for arbitrary values of $A$.

The problem reported above can be solved in different ways. The straightforward one is to use a coupling with higher order derivatives of the spin- $\frac{3}{2}$ field which explicitly involves the $\mathcal{P}_{\mu \nu}^{\frac{3}{2}}(p)$ projection operator:

$$
\frac{\mathrm{g}_{\Delta N \pi}}{m_{\pi} m_{N}^{4}} \bar{\psi}_{N}(x)\left[\square \mathcal{P}_{\mu \nu}^{\frac{3}{2}}(\partial) \Delta^{v}(x)\right] \partial^{\mu} \pi(x)+\text { h.c.. }
$$

The use of $\mathcal{P}_{\mu \nu}^{\frac{3}{2}}(\partial)$ ensures that only the spin- $\frac{3}{2}$ part of the propagator contributes and the d'Alembert-operator guarantees that no other singularities except the mass pole term $\left(p^{2}-m^{2}\right)^{-1}$ appear in the matrix element. Note, that the coupling written in the form of Eq. (10) restores the invariance of the full Lagrangian under the point-like transformations $\Delta_{\mu} \rightarrow \Delta_{\mu}+z \gamma_{\mu} \gamma^{v} \Delta_{v}$.

To keep the interaction term in the full Lagrangian as simple as possible we propose here another coupling

$$
\begin{aligned}
\mathcal{L}_{I} & =\frac{\mathrm{g}_{\Delta N \pi}}{m_{\pi} m_{N}} \\
& \times \bar{\psi}_{N}(x)\left[\Gamma_{v \eta}(A, \partial) \Delta^{\eta}(x)\right] \partial^{v} \pi(x)+\text { h.c., }(11)
\end{aligned}
$$

with the modified vertex operator $\Gamma_{v \eta}(A, \partial)$ depending on the parameter $A$ :

$$
\begin{aligned}
& \Gamma_{\nu \eta}(A, \partial)=\gamma_{5} \gamma^{\mu} \epsilon_{\mu \nu \rho \sigma} \theta_{\eta}^{\sigma}(A) \partial^{\rho}, \\
& \theta_{\sigma \eta}(A)=g_{\sigma \eta}-\frac{A+1}{2} \gamma_{\sigma} \gamma_{\eta} .
\end{aligned}
$$

In momentum space at $A=-1$ the vertex function Eq. (12) reduces to that suggested by Pascalutsa. The $\theta_{\mu \nu}(A)$ operator has a simple physical meaning: it relates the Rarita-Schwinger theory to the general case of arbitrary A. Hence, the R-S propagator, can be obtained from the general propagator by means of the transformation $G_{\mu \nu}^{\mathrm{RS}}(p)=$ $\theta_{\mu \rho}(A) G_{\rho \sigma}(p, A) \theta_{\sigma v}(A)$.

Using the coupling Eq. (11) the final result for the matrix element of $\pi N$ scattering is independent on the nonepole spin- $\frac{1}{2}$ terms in the full propagator

$$
\begin{array}{r}
{\left[\Gamma_{\mu \rho}\left(A, p_{\Delta}\right) G^{\rho \sigma}\left(p_{\Delta}, A\right) \Gamma_{\sigma \nu}^{\dagger}\left(A, p_{\Delta}\right)\right]} \\
=\frac{p+m}{p^{2}-m^{2}} \frac{p_{\Delta}^{2}}{m_{N}^{2}} \mathcal{P}_{\mu \nu}^{\frac{3}{2}}\left(p_{\Delta}\right)
\end{array}
$$

\footnotetext{
${ }^{1}$ We omit isospin indices.
} 
and coincides with that obtained for the case $A=-1$. The coupling Eq. (11) can be written in a more compact form which does not contain the Levi-Civita tensor explicitly

$$
\begin{aligned}
& \mathcal{L}_{I}=\frac{\operatorname{ig}_{\Delta N \pi}}{4 m_{\pi} m_{N}} \\
& \times \bar{\psi}_{N}(x)\left[\gamma^{\sigma \rho v} \theta_{\sigma \eta}(A) \partial^{\rho} \Delta^{\eta}(x)\right] \partial^{v} \pi(x)+\text { h.c., }(15)
\end{aligned}
$$

where $\gamma^{\sigma \rho v}=\left\{\gamma^{\sigma \rho}, \gamma^{\nu}\right\}$ and $\gamma^{\sigma \rho}=\left[\gamma^{\sigma}, \gamma^{\rho}\right]$ and $\theta_{\sigma \eta}(A)$ is defined in Eq. (13).

The full Lagrangian for the interacting $\Delta N \pi$ fields can be written in the form

$$
\mathcal{L}^{\frac{3}{2}}=\mathcal{L}_{0}^{\frac{3}{2}}+\mathcal{L}_{I}+\mathcal{L}_{0}^{\pi}+\mathcal{L}_{0}^{N},
$$

where $\mathcal{L}_{0}^{\pi}=\pi\left(\square+m^{2}\right) \pi$ and $\mathcal{L}_{0}^{N}=\bar{\psi}_{N}(i \not \partial-m) \psi_{N}$ stand for the free Lagrangians of pion and nucleon fields, respectively. The free spin- $\frac{3}{2}$ Lagrangian $\mathcal{L}_{0}^{\frac{3}{2}}$ and $\Delta N \pi$ coupling $\mathcal{L}_{I}$ are given by expressions Eq. (6) and Eq. (11). The Lagrangian Eq. (16) depends on one arbitrary parameter $A$ which points to the freedom in choosing the 'off-shell' content of the theory. Although $\mathcal{L}_{0}^{\frac{3}{2}}$ contains one free parameter the physical observables should not depend on it. Without going into the details, we mention that similar conclusion can be made for the electromagnetic coupling and refer to ref. [11] for details.

Summarizing this work, we could show that the gaugeinvariant $\Delta N \pi$ coupling, originally suggested by Pascalutsa for spin- $\frac{3}{2}$ fields, removes the off-shell degrees of freedom only for a specific choice of the spin- $\frac{3}{2}$ propagator but not in the general case. In the general case the spin- $\frac{3}{2}$ propagator contains a term associated with the $\left[\frac{1}{2} \otimes 1\right]_{\frac{1}{2}}$ irreducible representation. We have shown that the problem can be solved by introducing higher order derivatives to the interaction Lagrangian or by generalizing the original $\Delta N \pi$ coupling suggested by Pascalutsa. In the latter case the full Lagrangian of the interacting $\Delta N \pi$ fields depends on one free parameter which reflects the freedom in choosing an off-shell content of the theory.

\subsubsection{Gauge properties of spin-5/2 fields}

The description of pion- and photon-induced reactions in the resonance energy region is faced with the problem of proper treatment of higher spin states. In 1941 Rarita and Schwinger (R-S) suggested a set of equations which a field function of a higher spin should obey [10]. Another formulation has been developed by Fierz and Pauli [16] where an auxiliary field concept is used to derive subsidiary constraints on the field function. Regardless of the procedure used the obtained Lagrangians for free higherspin fields turn out to be always dependent on arbitrary free parameters. For the spin- $\frac{3}{2}$ fields this issue is widely discussed in the Literature (see e.g. $[11,17,18]$ for a modern status of the problem). The case of the spin- $\frac{5}{2}$ fields is even less studied. First attempts were made in [19, 20]. The authors of [20] deduced an equation of motion as a decomposition in terms of corresponding projection operators with additional algebraic constraints on parameters of the decomposition.
The free particle propagator is a central quantity in most of the calculations in quantum field theory. In [20] the authors deduced a spin- $\frac{5}{2}$ propagator written in operator form. In practical calculations, however, one needs an explicit expression of the propagator. An attempt to construct a propagator only from the spin- $\frac{5}{2}$ projection operator has been made in $[21,22]$. We demonstrated that such a quantity is not consistent with the equation of motions for the spin- $\frac{5}{2}$ field. In addition, hermiticity can be violated, as was pointed in [14]. Clearly, it is important to derive the propagator and investigate its properties in detail. To the best of our knowledge our study was the first attempt in that direction. Hence, the aim of the work was to deduce an explicit expression for the spin- $\frac{5}{2}$ propagator and study its properties. Guided by the properties of the free spin- $\frac{3}{2}$ Rarita-Schwinger theory one would expect the equation of motion for the spin- $\frac{5}{2}$ field has two arbitrary free parameters which define the non-pole spin- $\frac{3}{2}$ and $-\frac{1}{2}$ contributions to the full propagator. The coupling of the spin- $\frac{5}{2}$ field to the (e.g.) pion-nucleon final state is therefore defined up to two off-shell parameters which scale the non-pole contributions to the physical observables. Hence, one can ask whether such an arbitrariness can be removed from the theory.

The possibility to construct consistent higher-spin massless theories has been pointed out already a while ago by Weinberg and Witten [23]. As we demonstrated in [11] the demand for gauge-invariance may not be enough to eliminate the extra degrees of freedom at the interaction vertex. The problem appears when the theory does not have a massless limit. However, a coupling which removes non-pole terms from the spin- $\frac{5}{2}$ propagator can be easily constructed by using higher order derivatives. A corresponding interaction Lagrangian has been deduced in [11] for the case of spin- $\frac{3}{2}$ fields and can be easily extended to higher spins too, as exercised in [14].

The field function of higher spins in a spinor-tensor representation is a solution of the set of equations suggested by Rarita and Schwinger in [10]. In a consistent theory the description of the free field is specified by setting up an appropriate Lagrange function $\mathcal{L}\left(\psi_{\mu \nu}, \partial_{\rho} \psi_{\mu \nu}\right)$. The spin- $\frac{5}{2}$ Lagrangian in the presence of the auxiliary spinor field $\xi(x)$ can be written in the form

$$
\mathcal{L}=\mathcal{L}^{(1)}+\mathcal{L}^{(2)}+\mathcal{L}^{(\text {aux })}
$$

where the lengthy and mathematically involved expressions for the three pieces are found in Ref. [14]. An important observation is that the Lagrangian in Eq. (17) in general depends on only three independent real parameters $a, b$, and $c$.

By variations with respect to $\psi_{\mu \nu}$ and $\xi$ two equations of motion are obtained which in momentum space are given as

$$
\begin{gathered}
\left(\Lambda_{\mu v ; \rho \sigma}^{(1)}(p)+\Lambda_{\mu v ; \rho \sigma}^{(2)}(p)\right) \psi^{\rho \sigma}(p)+c m \mathrm{~g}^{\mu v} \xi(p)=0 \\
m c \mathrm{~g}^{\rho \sigma} \psi_{\rho \sigma}(p)+B(a, b, c)(p+3 m) \xi(p)=0
\end{gathered}
$$

where the explicit forms of the operators $\Lambda_{\mu v ; \rho \sigma}^{(1)}(p)$, $\Lambda_{\mu v ; \rho \sigma}^{(2)}(p)$ are found in [14]. Here, it is of interest that 
the operator $\Lambda_{\mu \nu ; \rho \sigma}^{(1)}(p)$ would give an equation of motion $\Lambda_{\mu v ; \rho \sigma}^{(1)}(p) \psi^{\mu v}=0$ for the spin- $\frac{5}{2}$ fields provided $\mathrm{g}^{\mu v} \psi_{\mu v}=0$, where the later property is assumed a priori. However, the corresponding inverse operator $\left[\Lambda_{\mu v ; \rho \sigma}^{(1)}(p)\right]^{-1}$ has additional non-physical poles in the spin- $\frac{1}{2}$ sector. This indicates that the constraint $\mathrm{g}^{\mu \nu} \psi_{\mu v}=0$ should also follow from the equation of motion and cannot be assumed a priori. The second operator $\Lambda_{\mu v ; \rho \sigma}^{(2)}(p)$ acts only in the spin- $\frac{1}{2}$ sector of the spin-tensor representation. This can be checked by a direct decomposition of the operator in terms of projection operators [14]. The same conclusion can be drawn from the observation that $\Lambda_{\mu v ; \rho \sigma}^{(2)}(p)$ is orthogonal to all $\mathcal{P}_{\rho \sigma ; \tau \delta}^{\frac{5}{2}}(p), \mathcal{P}_{i j ; \rho \sigma ; \tau \delta}^{\frac{3}{2}}(p)$ projection operators, where $i, j=1,2$. Hence the parameter $b$ is related only to the spin- $\frac{1}{2}$ degrees of freedom whereas $a$ scales both spin- $\frac{3}{2}$ and $-\frac{1}{2}$ ones.

Of particular interest for spectroscopic research is the coupling of resonances to meson-nucleon channels. In the case of the spin- $\frac{5}{2}$ field in the spinor-tensor representation we deal with a system $\left(\psi_{\mu \nu}, \xi\right)$ which contains auxiliary degrees of freedom. The question arises whether the nonphysical degrees of freedom could be eliminated from physical observables. Here we consider a simple case of spin- $\frac{5}{2}$ resonance contribution to $\pi N$ scattering. The corresponding $\pi N N_{\frac{5}{2}}^{*}$ coupling can be chosen as follows

$$
\begin{aligned}
\mathcal{L}_{I} & =\frac{\mathrm{g}_{\pi N N^{*}}}{4 m_{\pi}^{2}} \\
& \times\left(\bar{\psi}_{N}(x), 0\right) \Gamma_{\mu v ; \rho \sigma}\left[\mathfrak{P}\left(\begin{array}{c}
\psi^{\rho \sigma} \\
\xi
\end{array}\right)\right] \partial^{\mu} \partial^{v} \pi(x) \\
& + \text { h.c., }
\end{aligned}
$$

where the nucleon field is written as $\left(\bar{\psi}_{N}(x), 0\right)$ which implies the absence of auxiliary fields in the final state. The operator

$$
\mathfrak{P}=\left(\begin{array}{ll}
1 & 0 \\
0 & 0
\end{array}\right)
$$

projects out the spin- $\frac{5}{2}$ field and ensures that there is no coupling to $\xi$. Hence, only the spin- $\frac{5}{2}$ component of the propagator $G_{\mu v ; \rho \sigma}^{\frac{5}{2}}(p)$ contributes to physical observables at any order of perturbation theory. In [14] we could demonstrate that the inclusion of auxiliary degrees of freedom in the vector field does not affect the physical observables. To the best of our knowledge this statement is not generally proven for the $\left(\psi_{\mu \nu}, \xi\right)$ system beyond the perturbation expansion. The reason is that the equation of the motion for massive spin- $\frac{5}{2}$ field in the spinor-tensor representation is defined only in the presence of an auxiliary field. This is unlike the case of the vector field where auxiliary degrees of freedom can be removed by proper field transformations. These degrees of freedom contribute due to $\psi_{\mu v}-\xi$ mixing. The mixing takes place only between the spin- $\frac{1}{2}$ sector of the spinor-tensor and the auxiliary spinor fields. One may therefore hope that the use of a coupling which suppresses the spin- $\frac{1}{2}$ contributions would also prevent the appearance of the auxiliary degrees of freedom in the physical observables in the non-perturbative regime.
The solution to the problem is following closely the results for spin-3/2 fields, presented in [11]. According to our previous findings the interaction vertex fulfills the condition $\gamma \cdot \Gamma=\Gamma \cdot \gamma=0$. With this constraint one finds that only the $\mathcal{P}_{\mu v ; \rho \sigma}^{\frac{5}{2}}(q)$ projector fulfills the desired property [14], ensuring that only the spin- $\frac{5}{2}$ part of the propagator contributes. The formalism also guarantees that no other singularities except the mass pole term $\left(p^{2}-m^{2}\right)^{-1}$ appear in the amplitude. Thus, the physical observables no longer depend on the arbitrary parameters $a$ and $b$ of the free Lagrangian. Finally, the $\pi N$ scattering amplitude reads

$$
\begin{aligned}
& \mathcal{M}=\left(\frac{\mathrm{g}_{\pi N N^{*}}}{m_{\pi}^{2}}\right)^{2} \bar{u}_{N}\left(p^{\prime}\right) \\
& {\left[\left(\frac{q^{2}}{m_{R}^{2}}\right)^{4} \mathcal{P}_{\mu v ; \lambda \tau}^{\frac{5}{2}}(q)\right] u_{N}(p) k^{\prime \mu} k^{\prime v} k^{\lambda} k^{\tau},}
\end{aligned}
$$

Summarizing this part of the project, we have investigated the general properties of the free spin- $\frac{5}{2}$ fields in the spinor-tensor representation. We could show that the Lagrangian in general depends on three arbitrary parameters; two of them are associated with the lower spin- $\frac{3}{2}$ and $-\frac{1}{2}$ sector of the theory whereas the third one is linked to the auxiliary field $\xi$. We have deduced a free propagator of the system in form of a $2 \times 2$ matrix in the $\left(\psi_{\mu \nu}, \xi\right)$ space. The diagonal elements stand for the propagation of the spin- $\frac{5}{2}$ and $\xi$ fields whereas the non-diagonal ones correspond to $\psi_{\mu \nu}-\xi$ mixing. The mixing takes place between the spin$\frac{1}{2}$ sector of the spinor-tensor representation and an auxiliary spinor field. An important result was that the auxiliary degrees of freedom do not contribute to the physical observables calculated within the perturbation theory provided there is no coupling to $\xi$. As an application to hadron spectroscopy, the $\pi N N_{\frac{5}{2}}^{*}$ interaction vertex was discussed. Gauge invariance was obtained by constructing a coupling with higher order derivatives. In the latter case the amplitude of the $\pi N$ scattering does not depend on the arbitrary parameters of the free Lagrangian. The suggested coupling is generalized to the Rarita-Schwinger fields of any half-integer spin.

\section{Strangeness production on the nucleon}

Strangeness production on the nucleon by excitation of resonances which decay into kaon-hyperon channels is an important spectroscopic tool giving access to the SU(3) flavour structure of baryons. Moreover, such exotic channels like the kaon-hyperon final states are expected to play a cental role in identifying hitherto undetected excited states of the nucleon, thus addressing the still open question of the notorious problem of missing resonances. In [5] we have performed a study of the pion- and photoninduced $K \Lambda$ reactions within our unitary coupled-channel effective Lagrangian approach. A major goal of those investigations was to address the at that time still open question on the major contributions to the associated strangeness production channels. Since $K \Lambda$ photoproduction data $[24,25]$ gave an indication for missing resonance 
contributions, a combined analysis of the $(\pi, \gamma) N \rightarrow K \Lambda$ reactions was expected to identify clearly these states. Assuming small couplings to $\pi N$, these hidden states should not exhibit themselves in the pion-induced reactions and, consequently, in the $\pi N \rightarrow K \Lambda$ reaction. The aim of our calculations was to explore to what extent the data available at that time can be explained by known reaction mechanisms without introducing new resonances. Our results for total cross sections are displayed in Fig.3 and further results on differential cross sections, polarization observables and angular distributions are found in [5]. As discussed in [5] the SAPHIR [24] and the CLAS [25] data sets, in fact, are leading to two slightly different sets of interaction parameters, reflecting and emphasizing the differences among the two measurements. Below, that point is discussed again.

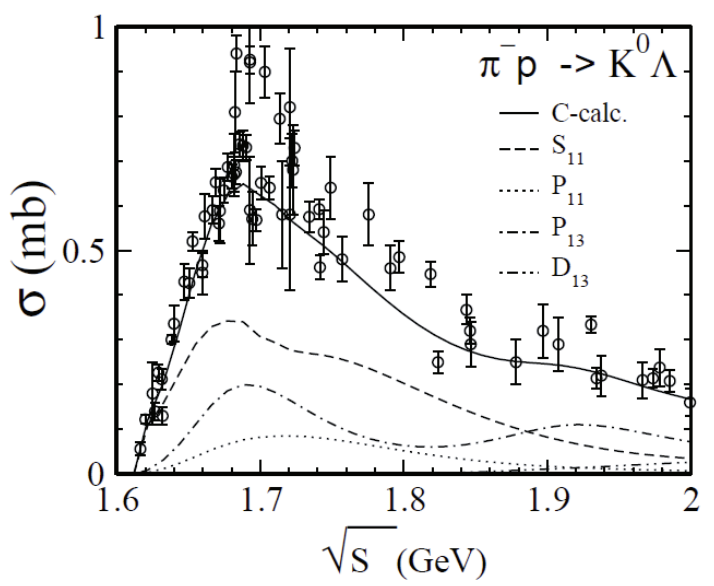

Figure 3. $\pi^{-} p \rightarrow K^{0} \Lambda$ total partial wave cross sections, predicted by parameter set $C$ of Ref. [5], obtained from a fit to the CLAS data [25]. The experimental cross section data are taken from [26-28].

More recent CLAS-data on $K \Sigma$ production inspired us to a revised updated large scale coupled-channels analysis of associated strangeness production on the nucleon. Based on the coupled-channel effective Lagrangian formalism underlying the Giessen model (GiM) a combined analysis of $(\pi, \gamma) N \rightarrow K \Sigma$ hadro- and photo-production reactions were performed. The analysis covered a centerof-mass energy range up to $2 \mathrm{GeV}$. The recent photoproduction data obtained by the CLAS, CBELSA, LEPS, and GRAAL groups were included into our calculations. The central aim was to extract the resonance couplings to the $K \Sigma$ state. In [6] the Giessen model was used to reanalyze newly released data from various experimental groups for $K \Sigma$ production on the nucleon. Both s-channel resonances and t,u-channel background contributions are found to be important for an accurate description of angular distributions and polarization observables, assuring a high quality description of the data. The extracted properties of isospin $I=3 / 2$ resonances were discussed in detail. We found that the $I=1 / 2$ resonances are largely determined by the non-strangeness channels.

Our calculations included 11 isospin $I=1 / 2$ resonances and 9 isospin $I=3 / 2$ resonances, respectively. In this work we continued the investigations of the $I=1 / 2$ and $3 / 2$ sectors with the parameters fitted to newly published $K \Sigma$ photoproduction data together with the previous $\pi N \rightarrow K \Sigma$ measurements in the energy region $\sqrt{s} \leq 2.0 \mathrm{GeV}$. The included $K \Sigma$ photoproduction data are those of the $\gamma p \rightarrow K^{+} \Sigma^{0}$ published by the LEPS [2931], CLAS [32, 33] and GRAAL [43] group, and those of $\gamma p \rightarrow K^{0} \Sigma^{+}$released by the CLAS [44] and CBELSA [45] collaboration, respectively. The SAPHIR data have been left out here because of the known inconsistencies of the $K^{+} \Sigma^{0}$ data [24] with the corresponding CLAS and GRAAL data (for the details, see Ref. [33]). Also, the $K^{0} \Sigma^{+}$SAPHIR data [24] have much bigger error bars than those of the CBELSA and CLAS group. Here, the data before 2002 are also no longer used.

Strangeness production on the nucleon plays a key role for our understanding of baryon structure and elementary reactions with hadrons. In addition, such production reactions are also an appropriate tool to identify excited states $N^{*}$ of the nucleon which decay preferentially into hyperon-kaon channels, thus adding to solve the problem of missing resonances. In [6] we have analysed the latest CLAS-, CBELSA-data sets and re-analysed the earlier SAPHIR-data on photoproduction of kaons on the nucleon. The Giessen model was used, describing meson production on the nucleon by a coupled channels $\mathrm{K}$-matrix approach including meson production by photoproduction and pion-induced reactions as initial channels. The Giessen model is obeying the elementary symmetries of hadron physics and conserving unitarity. Meson production proceeds through s-channel resonances and t- and $\mathrm{u}$-channels re-scattering processes. Results for total cross sections are shown in Fig.4. Up to a total center-of-mass energy of about $\sqrt{s}=2 \mathrm{GeV}$ the data are well described.

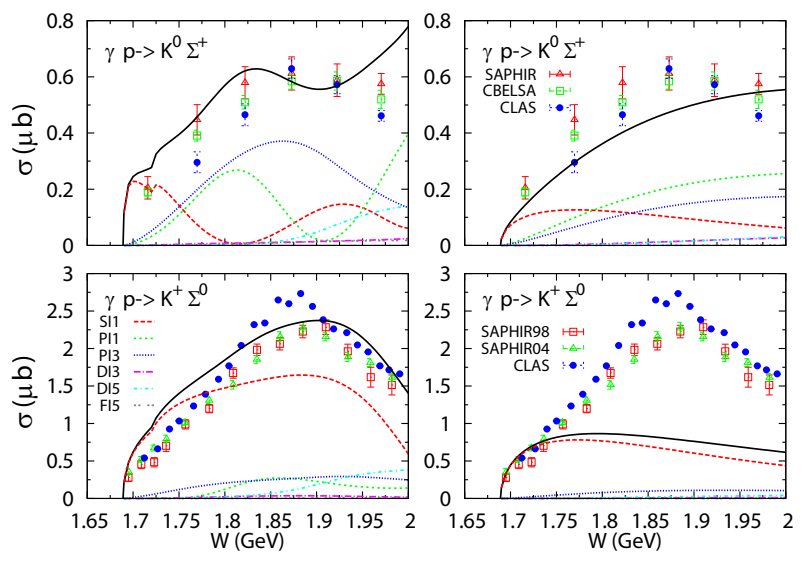

Figure 4. Total cross sections for kaon production on the nucleon. Results of the Giessen model [6] are compared to CLAS, CBELSA, and SAPHIR data.

The analysis included all charge channels, $K^{ \pm} \Sigma^{\mp}$ and $K^{0} \Sigma^{0}$. We achieved a quite satisfactory description of the $\gamma p \rightarrow K^{+} \Sigma^{0}$ data $\left(\chi^{2}=1.8\right)$ and the $\gamma p \rightarrow K^{0} \Sigma^{+}$data $\left(\chi^{2}=2.0\right)$. However, the pion-induced strangeness production reactions are described slightly less accurate as indicated by the corresponding $\chi^{2}$ values of $\chi^{2}=4.1,3.2$ and 


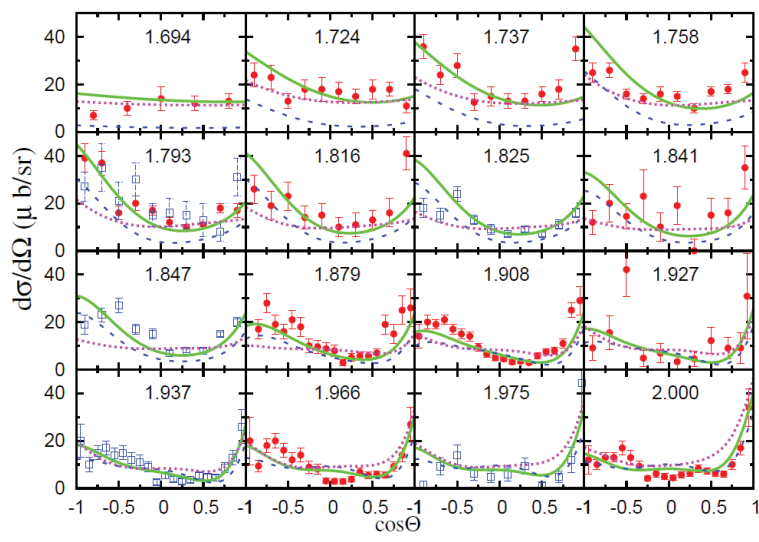

Figure 5. The differential cross section of $\pi^{-} p \rightarrow K^{0} \Sigma^{0}$ reaction. The solid (green), dashed (blue) and dotted (magenta) lines are the full model calculation, the model calculation with the $S_{11}(1650)$ and $F_{15}(1680)$ turned off, respectively. The numerical labels denote the center of mass energies in units of $\mathrm{GeV}$. CBELSA and CLAS data are shown for comparison.

2.8 for the $\pi^{+} p \rightarrow K^{+} \Sigma^{+}, \pi^{-} p \rightarrow K^{0} \Sigma^{0}$ and $\pi^{-} p \rightarrow K^{+} \Sigma^{-}$ reactions, respectively. The parameters have been varied in our fit simultaneously to the $I=1 / 2$ and $3 / 2$ sectors. Because of the smallness of the $N^{*} K \Sigma$ couplings, all previously obtained Breit-Wigner masses, branching ratios and couplings corresponding to non-strangeness production [2] are hardly affected by the additional $K \Sigma$ photoproduction data, so we could concentrate on the properties of the $I=3 / 2$ resonances. Although the new data are available with reduced total errors the refitted model parameters were changed only very little. A typical result is displayed in Fig. 5, illustrating the quality of the description on the example of $\pi^{-} p \rightarrow K^{0} \Sigma^{0}$ reaction. The complete set of results, including partial wave cross sections, angular distributions of cross sections and polarization observables for the full set of $K \Sigma$ exit channels are found in [6].

\section{$5 \eta$-meson production}

Understanding the dynamics of eta-meson production and, vice versa, the decay of nucleon resonances into the nucleon-eta exit channel is of ongoing interest in hadron spectroscopy. The $\eta$-meson photoproduction on the proton has been measured with high precision by the Crystal Ball collaboration at MAMI [34]. These high-resolution data provide a new step forward in understanding the reaction dynamics and in the search for a signal from the 'weak' resonance states. The main result reported in [34] is a very clean signal for a dip structure around $W=1.68 \mathrm{GeV}$, seemingly confirming older data [35-38]. This raised the question on the origin of that structure, eventually indicating the appearance of a new narrow, possibly exotic, resonance state.

The aim of the study was to extend our previous coupled-channels analysis of the $\gamma p \rightarrow \eta p$ reaction by including the data from the new high-precision measurements [34]. The main question is whether the $\eta p$ reaction dynamics can be understood in terms of the established resonance states or whether a new state has to be introduced, thus confirming previous conjectures. A major issue for the analysis is unitarity and a consistent treatment of self-energy effects as visible in the total decay width of resonances. Since the latter are driven by hadronic interactions the analysis of photo-production data requires the knowledge of the hadronic transition amplitudes as well. Hence, a coupled-channels description as in the Giessen model is an indispensable necessity.

As discussed in very detail in [4] various relevant meson-baryon coupling constants were newly determined at the occasion of this work in large scale coupledchannels calculations. This gave rise to improved constraints on the interaction parameters and the derived resonance parameters, i.e. masses and widths. As an representative example we mention here the mass and width of the $D_{13}(1520)$ resonance. Our results confirm the values obtained by Arndt et al. [40]: mass $M=1516 \pm 10 \mathrm{MeV}$ and width $\Gamma=106 \pm 4 \mathrm{MeV}$, respectively. It is interesting to note that the mass of this resonance deduced from pion photoproduction tends to be $10 \mathrm{MeV}$ lower that the values derived from the pion-induced reactions [41]. The second $D_{13}(1900)$ state has a very large decay width. We associate this state with the $D_{13}(2080)$ two-star state, proposed by PDG. The GiM results for the $\eta$-photo production channel are shown in Fig. 6 together with the experimental data.

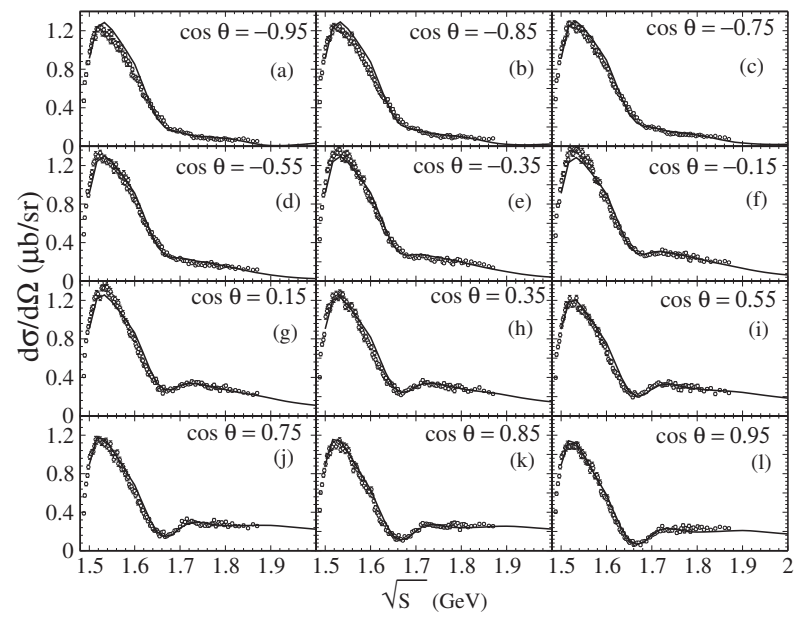

Figure 6. Differential $\eta p$ cross section compared to MAMI data [34].

We obtain a very satisfactory agreement with the experimental data in the whole kinematical region. The first peak is related to the $S_{11}(1535)$ resonance contribution. Similar to the $\pi^{-} p \rightarrow \eta n$ reaction the $S_{11}(1650)$ and $S_{11}(1650)$ states interfere destructively producing a dip around $\mathrm{W}=1.68 \mathrm{GeV}$. The coherent sum of all partial waves leads to the more pronounced effect from the dip at forward angles. We also corroborate our previous findings [3] where a small effect from the $\omega N$ threshold was found.

We also do not find any strong indication for contributions from a hypothetic narrow $P_{11}$ state with a width of $15-20 \mathrm{MeV}$ around $\mathrm{W}=1.68 \mathrm{GeV}$. It is natural to assume that the contribution from this state would induce a strong modification of the beam asymmetry for energies close to 
the mass of this state. This is because the beam asymmetry is less sensitive to the absolute magnitude of the various partial wave contributions but strongly affected by the relative phases between different partial waves. Thus even a small admixture of a contribution from a narrow state might result into a strong modification of the beam asymmetry in the energy region of $\mathrm{W}=1.68 \mathrm{GeV}$.

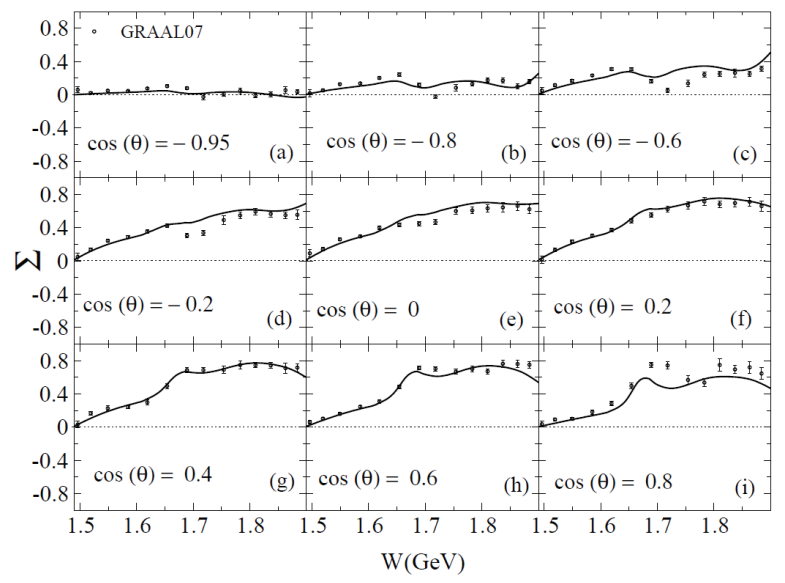

Figure 7. (Differential $\eta p$ cross section compared to GRAAL data [38].

In Fig. 7 we show results for the photon-beam asymmetry $\Sigma$ in comparison with the GRAAL data. One can see that even close to the $\eta N$ threshold where our calculations exhibit a dominant $S_{11}$ production mechanism the beam asymmetry is non-vanishing for angles $\cos (\theta) \geq$ -0.2 . This shows that this observable is very sensitive to very small contributions from higher partial waves. At $\mathrm{W}=1.68 \mathrm{GeV}$ and forward angles the GRAAL measurements show a rapid change of the asymmetry behavior. We explain this effect by a destructive interference between the $S_{11}(1535)$ and $S_{11}(1650)$ resonances which induces the dip at $\mathrm{W}=1.68 \mathrm{GeV}$ in the $S_{11}$ partial wave. The strong drop in the $S_{11}$ partial wave modifies the interference between $S_{11}$ and other partial waves and changes the asymmetry behavior. Note that the interference between $S_{11}(1535)$ and $S_{11}(1650)$ and the interference between different partial waves are of different nature. The overlapping of the $S_{11}(1535)$ and $S_{11}(1650)$ resonances does not simply mean a coherent sum of two independent contributions, but also includes rescattering (coupled-channel effects). Such interplay is hard to simulate by the simple sum of two Breit-Wigner forms since it does not take into account rescattering due to the coupled-channel treatment.

\section{Double-pion production on the nucleon}

In certain energy regions the $\pi N \rightarrow 2 \pi N$ reaction accounts for up to $50 \%$ of the $\pi N$ inelasticity as seen in Fig.1. Therefore, this production channel had been included from the very beginning into the GiM approach, albeit in a schematic manner. An improved and considerably extended description of double-pion production within our coupled channels scheme was started recently and first results are found in [7]. The inclusion of multi-meson con-

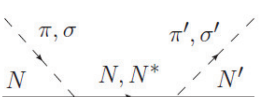

(a)

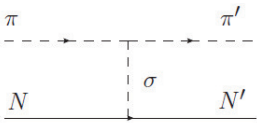

(c)
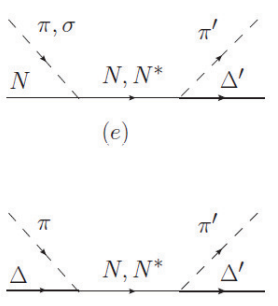

$(g)$

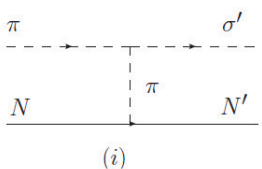

Figure 8. Diagrammatic structure of the tree-level interactions contributing to double-pion production on the nucleon.

figurations into a coupled channels approach is a highly non-trivial exercise in three-body dynamics and beyond. In view of the complexities physically meaningful approximations are necessary, retaining the essential dynamical aspects but making numerical calculations feasible. For that goal the ansatz used in [7] relies on an isobar description of intermediate two-pion configurations and their decay into the final double pion states on the mass shell. In Fig. 8 the tree-level interactions for two-pion production are displayed diagrammatically. The derived processes contributing to the T-matrix of double-pion production on the nucleon in that energy region are depicted in Fig.9.

This approach allows for the direct analysis of the $2 \pi N$ experimental data. Since the corresponding Dalitz plots are found to be strongly non-uniform it is natural to assume that the main effect to the reaction comes from the resonance decays into isobar subchannels [42]. The most important contributions are expected to be from the intermediate $\sigma N, \pi \Delta_{1232}$, and $\rho N$ states. The analysis of the $\pi N \rightarrow 2 \pi N$ reaction would therefore provide very important information about the resonance decay modes into different isobar final states. Presently, lattice simulations, e.g. [47, 48], and functional approaches as in [49] are applied to approach the baryon spectrum from the QCD-side. However, despite considerable progress a number of open problems are persisting like the incorporation of the dispersive self-energies from the coupling of the QCD configurations to the meson-nucleon decay channels. Similar to the constituent quark models $[50,51]$ the QCD-inspired 

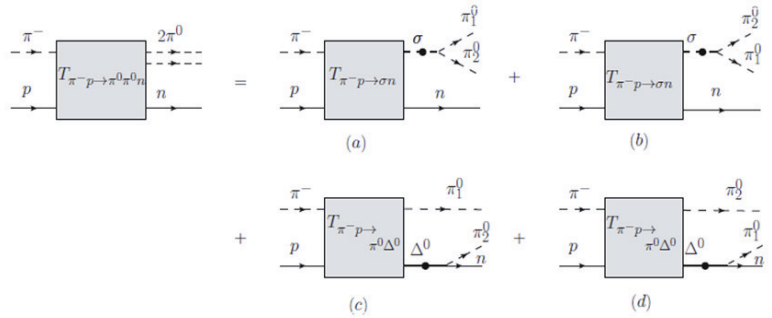

Figure 9. The processes contributing to double-pion production T-matrix are depicted diagrammatically: (a) and (b) production through the $\sigma$-isobar, (c) and (d) production through the $\Delta^{0}$-isobar. Symmetrization is indicated.

calculations predict a much richer baryon spectrum [48] than observed experimentally so far.

Clearly, an unambiguous identification of the excitation spectrum of baryons would provide an important link between theory and experiment. On the experimental side most of the non-strange baryonic states have been identified from the analysis of elastic $\pi N$ data [40, 52, 53]. However, as pointed out by Isgur [50], the signal of excited states with a small $\pi N$ coupling is likely to be suppressed in elastic $\pi N$ scattering. As a solution to this problem a series of photoproduction experiments has been done in order to accumulate enough data for detailed studies of the nucleon excitation spectrum. However, the results from the photoproduction reactions are still controversial. While recent investigations of the photoproduction reactions presented by the BoGa group [54] reported indications for some new resonances not all of these states are found in other calculations [41]. This raises a question about an independent confirmation of the existence of such states by other reactions. Because of the smallness of the electromagnetic couplings the largest contribution to the resonance self-energy comes from the hadronic decays. If the $N^{*} \rightarrow \pi N$ transition is small one can expect sizable resonance contribution into remaining hadronic decay channels. As a result the effect from the resonance with a small $\pi N$ coupling could still be significant in the inelastic pion-nucleon scattering: here the smallness of resonance coupling to the initial $\pi N$ states could be compensated by the potentially large decay branching ratio to other different inelastic final states. Such a scenario is realized e.g. in the case of the well known $N^{*}(1535)$ state. While the effect from this resonance to the elastic $\pi N$ scattering is only moderate at the level of total cross section its contribution to the $\pi N \rightarrow \eta N$ channel turns out to be dominant [4]. Since the $\pi N \rightarrow 2 \pi N$ reaction could account for up to $50 \%$ of the total $\pi N$ inelasticity this channel becomes very important not only for the investigation of the properties of already known resonances but also for the search for the signals of possibly unresolved states.

Another important issue in studies of the $2 \pi N$ channel is related to the possibility to investigate cascade transitions like $N^{* \prime} \rightarrow \pi N^{*} \rightarrow \pi \pi N$, where a massive state $N^{* \prime}$ decays via intermediate excited $N^{*}$ or $\Delta^{*}$. It is interesting to check whether such decay modes are responsible for the large decay width of higher lying mass states. So far only the $\pi N^{*}(1440)$ isobar channel has been considered in [42] in a partial wave analysis (PWA) of the $\pi N \rightarrow 2 \pi N$ experimental data [42].

There are several complications in the coupledchannel analysis of $2 \rightarrow 3$ transitions. The first one is the difficulty to perform the partial-wave decomposition of the three-particle state. The second complication is related to the issue of three-body unitarity. For a full dynamical treatment of the $2 \rightarrow 3$ reaction the Faddeev equations have to be solved. This makes the whole problem quite difficult for practical implementations. Here we address both issues and present a coupled-channel approach for solving the $\pi N \rightarrow 2 \pi N$ scattering problem in the isobar approximation. In this formulation the $(\pi / \pi \pi) N \rightarrow(\pi / \pi \pi) N$ coupled-channel equations are reduced to the two-body scattering equations for isobar production. Such a description accounts by construction for the full spectroscopic strength of intermediate channels and, in addition provides a considerable numerical simplification. Three-body unitarity leads to a relation between the imaginary part of the elastic scattering amplitude and the sum of the total elastic and inelastic cross sections by the well known optical theorem. Since in the isobar approximation the pions in the $\pi \pi N$ channel are produced from the isobar subchannels all contributions to the total $\pi N \rightarrow \pi \pi N$ cross section are driven by the isobar production. The optical theorem can be fulfilled if all discontinuities in isobar subchannels are taken into account. In the present work the three-body unitarity is maintained up to interference term between the isobar subchannels.

As a first application of our model we apply the developed approach for the study of the $\pi^{-} p \rightarrow \pi^{0} \pi^{0} n$ data in the first resonance energy region assuming the dominant $S_{11}$ and $P_{11}$ partial wave contributions in the $\sigma N$ and $\pi \Delta$ reaction subchannels. The main purpose of this paper is to introduce the model and demonstrate the feasibility of treating two-pion dynamics in the framework of a largescale coupled channels approach. For this aim, we restrict the calculations to the $\pi^{0} \pi^{0} n$ channel, taking advantage of the fact that only isoscalar two-pion and $\pi \Delta$ isobar channels are contributing to the process. We emphasize that this restriction is not a matter of principle but is only for the sake of a feasibility study. In particular, this means that at this stage we do not consider the $\rho N$ state but postpone its inclusion into the numerical scheme to a later stage. Naturally, the results presented in the following are most meaningful for the energy region of the $N^{*}(1440)$ Roper resonance.

The first resonance energy region is of particular interest because of the sizable effect from $N^{*}(1440)$. The dynamics of the Roper resonance turns out to be rich because of the two-pole structure reported in earlier studies $[55,56]$, (see $[40,57,58]$ for the recent status of the problem. ) The origin of the Roper resonance is also controversial. For example the calculations in the Jülich model explain this state as a dynamically generated pole due to the strong attraction in the $\sigma N$ subchannel. At the same time the Crystal Ball collaboration finds no evidence of strong $t$-channel sigma-meson production in their $\pi^{0} \pi^{0}$ data [59]. 
From the further analysis of the $\pi^{0} \pi^{0}$ production the effect of the sigma meson was found to be small [60]. On other hand the $p p \rightarrow p p \pi^{0} \pi^{0}$ scattering experiment by CELSIUS-WASA collaboration [61] finds the $\sigma N$ decay mode of the Roper resonance to be dominant.

In view of these problems we have performed an analysis of the $\pi^{0} \pi^{0}$ data assuming dominant contributions from the $S_{11}$ and $P_{11}$ amplitudes in the isospin $I=\frac{1}{2}$ channel. Since the effect from $N^{*}(1520)$ is expected to be important above $1.46 \mathrm{GeV}$ we have limited the present calculations up to $\sqrt{s}=1.46 \mathrm{GeV}$ energy region.

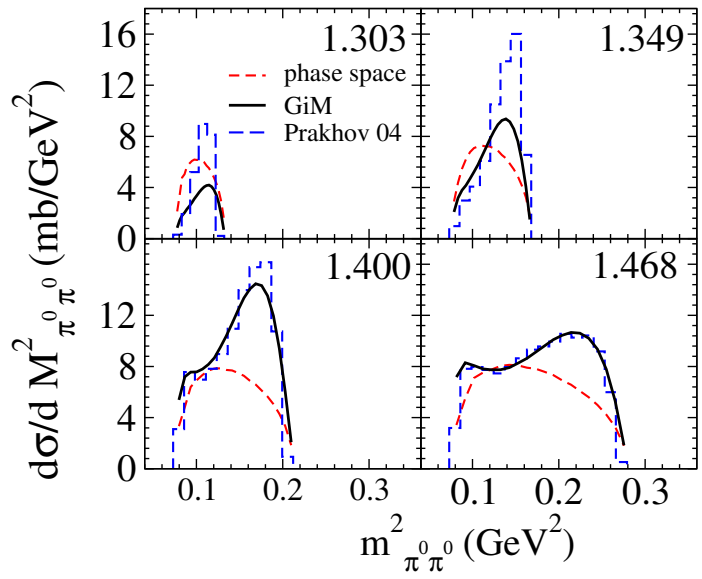

Figure 10. Reaction $\pi^{-} p \rightarrow \pi^{0} \pi^{0} n$ : differential cross section and bare phase space distribution (short dashed) as a function of $m_{\pi \pi}^{2}$ at fixed c.m. energies are compared to the Crystal Ball data (dashed) [60].

The difference between $\sigma N$ and $\pi \Delta$ production mechanism is seen in the invariant mass distributions, Fig. 10. Close to threshold the Crystal Ball data demonstrate a shift to the higher invariant masses for all energies up to 1.5 $\mathrm{GeV}$ whereas the three-body phase space tends to have a maximum at lower $m_{\pi^{0} \pi^{0}}^{2}$. In the present calculations the main contributions to the $\pi^{-} p \rightarrow \pi^{0} \pi^{0} n$ reaction close to threshold are driven by $t$-channel pion exchange. This mechanism produces the invariant distributions which are shifted to the higher $\pi^{0} \pi^{0}$ invariant masses. However, the present calculations do not completely follow the experimental data at 1.303 and $1.349 \mathrm{GeV}$.

In the region of the Roper resonance our calculations are able to describe the mass distributions rather satisfactorily. Also in this region the production strength is shifted to higher invariant masses $m_{\pi^{0} \pi^{0}}^{2}$. At the same time a peak at small $m_{\pi^{0} \pi^{0}}^{2}$ becomes also visible. In the present calculations the fit tends to decrease the magnitude of the $\pi \Delta_{1232}$ production and compensate it by enhancing the strength into $\sigma N$. The obtained decay branching ratio of $N^{*}(1440)$ for the $\sigma N$ channel is about twice as large as for the $\pi \Delta_{1232}$.

Both the small peak at small and the broad structure at large invariant masses are well reproduced indicating an important interplay between the $\sigma N$ and $\pi \Delta_{1232}$ production mechanism. It is interesting that the isoscalar correlations in the $\pi \pi$ rescattering are also found to be necessary in order to reproduce the asymmetric shape of the mass

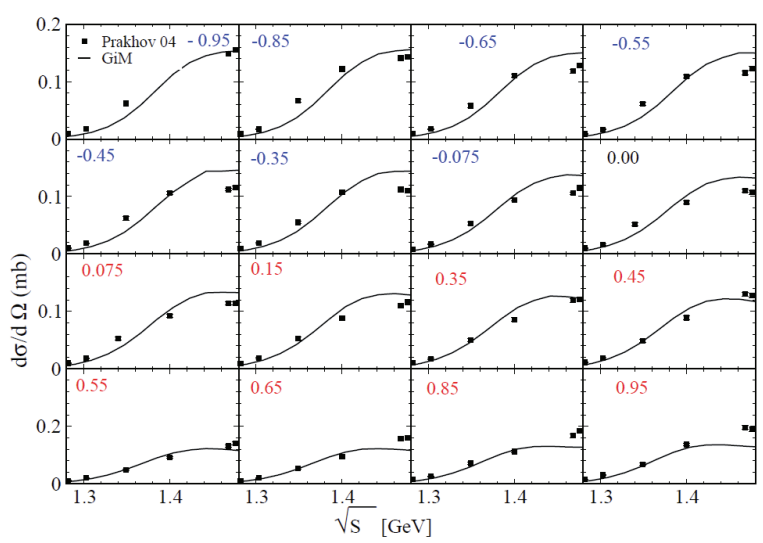

Figure 11. $\pi^{0} \pi^{0}$ differential cross sections for the reaction $\pi^{-} p \rightarrow \pi^{0} \pi^{0} n$ at fixed $t_{\pi^{0} \pi^{0}}=\cos \theta_{\pi^{0} \pi^{0}}$, shown in the upper left corner of the the panels. Energy distributions for $-0.95 \leq t_{\pi^{0} \pi^{0}} \leq$ +0.95 are shown. The experimental data are from [60].

distributions. Though the $\pi \Delta_{1232}$ production gives rise to a two-peak structure only the first one at small $m_{\pi^{0} \pi^{0}}^{2}$ is visible at energies 1.4-1.468 GeV. Within the present calculation the second peak at high $m_{\pi^{0} \pi^{0}}^{2}$ is not seen because of the large $\sigma N$ contributions. In the present study $\pi^{0} \pi^{0} n$ production is calculated as a coherent sum of isobar contributions. Though the interference effect are important they are found to be very small at the level of the total cross sections.

We briefly discuss the reaction data base used in the calculations. To simplify the analysis the $S_{11}$ and $P_{11} \pi N$ partial waves are directly constrained by the single energy solutions (SES) derived by GWU(SAID) [40]. The experimental data on the $\pi^{-} p \rightarrow \pi^{0} \pi^{0} n$ reaction are taken from [60]. These measurements provide high statistics data on the angular distributions $\mathrm{d} \sigma / d \Omega_{\pi \pi}$, where $\Omega_{\pi \pi}$ is the scattering angle of the $\pi \pi$ pair (or the final nucleon in c.m.). This data are accompanied by the corresponding statistical and systematical errors. No such information is available for the mass distributions in [60]. These observables are provided in a form of weighted events without systematic and statistical uncertainties. In the data analysis we impose the constraint that the integrated distributions must reproduce the total cross section of the $\pi^{-} p \rightarrow 2 \pi^{0} n$ reaction. We also have assigned about $10 \%$ error bars to each mass bin to perform the $\chi^{2}$ minimization. Starting from $1.46 \mathrm{GeV}$ the excitation of $N^{*}(1520)$ starts to be important. Already at this energy a small contribution from the spin $J=\frac{3}{2}$ partial wave could modify the angular and mass distributions. Because of this reason we do not try to fit the data above $1.46 \mathrm{GeV}$.

The calculated $\pi^{0} \pi^{0}$ differential cross sections are shown in Fig. 11 and compared to the Crystal Ball data as a function of the c.m. energy. The measurements demonstrate a rapid rise of the cross sections at the energies 1.3$1.46 \mathrm{GeV}$. We identify this behavior as an indication for the strong contribution coming from the Roper resonance. Indeed, the resulting $\pi N$ inelasticities from the GWU(SAID) [40] analysis indicate that the $P_{11}$ partial wave dominates 


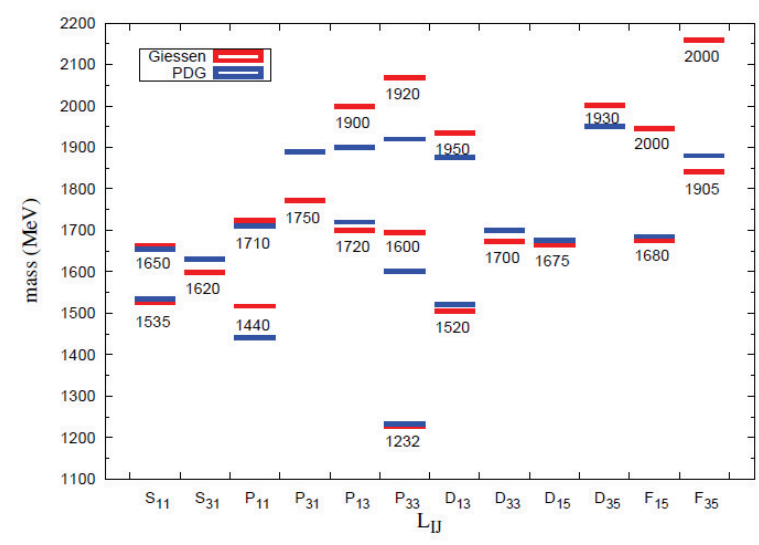

Figure 12. (Color online) Comparison of the GiM $N^{*}$ resonance level scheme in various partial waves to the recent PDG compilation [41].

the inelastic transitions at these energies. The inelasticity from the $S_{31}$ channel is about three times less than that from $P_{11}$. At the same time the $\Delta(1620)$ is strongly coupled to the $2 \pi N$ final state through the $\pi \Delta_{1232}$ decay [41]. Since the contribution from the $\sigma N$ subchannel is found in the present work to be about twice as large than that of $\pi \Delta_{1232}$ possible effects from the $\Delta(1620)$ resonance can safely be neglected in first approximation. We also allow the $N^{*}(1535)$ resonance decays to the $\pi \Delta_{1232}$ and $\sigma N$ isobar final states which are however found to be negligible. At energies close to $1.5 \mathrm{GeV}$ the obtained cross section slightly overestimates the experimental data at backward and underestimates them at forward scattering angles. This is a region where the $N^{*}(1520)$ starts to play a dominant role. We conclude that the contribution from the $D_{13}$ partial wave should be included for the successful description of the data at $1.5 \mathrm{GeV}$.

The invariant $\pi^{0} \pi^{0}$ mass distributions play a crucial role in the separation of the isobar contributions. The $\pi^{-} p \rightarrow \pi^{0} \pi^{0} n$ reaction close to threshold is dominated by the $\sigma N$ production due to the $t$-channel pion exchange. The nucleon Born term contribution to the $\pi \Delta_{1232}$ channel is found to be less significant. For the decay branching ratios of $N^{*}(1440)$ we obtain $R_{\sigma N}^{N(1440)}=27_{-9}^{+4} \%$ and $R_{\pi \Delta_{1232}}^{N(1440)}=12_{-3}^{+5} \%$.

The comparison of our results with the parameters extracted by the BoGa group $R_{\sigma N}^{N(1440)}=17_{-7}^{+7} \%$ and $R_{\pi \Delta_{1232}}^{N(1440)}=21_{-8}^{+8} \%$ demonstrates that despite on the visible difference in the central values these quantities could still coincide within their error bars. The extended analysis of the $\pi \pi N$ which includes higher partial waves would help to reduce the uncertainties of the extracted resonance properties.

\section{Summary}

Excited states of the nucleon as observed in $\pi N$ and $\gamma N$ reactions have been described in the GiM coupled channels approach. The theoretical background was discussed. The GiM approach is based on a phenomenological field theory described the baryons, mesons, and their interactions by a Lagrangian density conserving by construction the fundamental symmetries of QCD, including also chiral symmetry. Resonance and background contributions are generated consistently out of the tree-level interactions obtained from the Lagrangian. The scattering amplitudes are determined by linear system of coupled equations which is solved numerically in K-matrix approximation and partial wave representation. The gauge-invariant description of high-spin resonances was discussed in detail. Applications to selected reaction channels have been presented, ranging from single pion, eta, and kaon production to double-pion production. The spectroscopic results are summarized in Fig. 12 where the GiM spectrum of resonances is compared to the recent PDG resonance compilation.

The work reported here would not have been possible without the support from the Deutsche Forschungsgemeinschaft within the SFB/TR16 and the major contributions by Vitaliy Shklyar, and $\mathrm{Xu}$ Cao, now at IMP Lanzhou and CAS Beijing.

\section{References}

[1] V. Shklyar, G. Penner, and U. Mosel, Eur. Phys. J. A21, 445 (2004), nucl-th/0403064.

[2] V. Shklyar, H. Lenske, U. Mosel and G. Penner, Phys. Rev. C 71, 055206 (2005) Erratum: [Phys. Rev. C 72, 019903 (2005)] doi:10.1103/PhysRevC.72.019903, 10.1103/PhysRevC.71.055206 [nucl-th/0412029].

[3] V. Shklyar, H. Lenske, and U. Mosel, Phys.Lett. B650, 172 (2007), nucl-th/0611036.

[4] V. Shklyar, H. Lenske and U. Mosel, "Eta-meson production in the resonance energy region," Phys. Rev. C 87, 015201 (2013).

[5] V. Shklyar, H. Lenske and U. Mosel, Phys. Rev. C 72, 015210 (2005) doi:10.1103/PhysRevC.72.015210 [nucl-th/0505010].

[6] X. Cao, V. Shklyar, and H. Lenske, Phys.Rev. C88, 055204 (2013), 1303.2604.

[7] V. Shklyar, H. Lenske and U. Mosel, Phys. Rev. C 93, no. 4, 045206 (2016)

[8] B.C. Pearce and B.K. Jennings, Nucl. Phys. A528 (1991) 655.

[9] T. Feuster and U. Mosel, Phys. Rev. C584571998; T. Feuster and U. Mosel, ibid. 59, 460 (1999).

[10] W. Rarita and J. S. Schwinger, Phys. Rev. 60, 61 (1941).

[11] V. Shklyar and H. Lenske, Phys. Rev. C80, 058201 (2009).

[12] V. Pascalutsa and R. Timmermans, Phys. Rev. C60, 042201 (1999), nucl-th/9905065.

[13] V. Pascalutsa, Phys. Lett. B503, 85 (2001), hepph/0008026.

[14] V. Shklyar, H. Lenske and U. Mosel, Phys. Rev. C 82, 015203 (2010).

[15] M. Benmerrouche, R. M. Davidson, and N. C. Mukhopadhyay, Phys. Rev. C39, 2339 (1989). 
[16] M. Fierz and W. Pauli, Proc. Roy. Soc. Lond. A173, 211 (1939).

[17] V. Pascalutsa, Phys. Rev. D58, 096002 (1998), hepph/9802288.

[18] H. Krebs, E. Epelbaum and U.G. Meißner, Phys. Rev. C 80, 028201 (2009) doi:10.1103/PhysRevC.80.028201 [arXiv:0812.0132 [hep-th]].

[19] J. S. Schwinger, PARTICLES, SOURCES AND FIELDS, Reading 1973, 459p.

[20] F. A. Berends, J. W. van Holten, P. van Nieuwenhuizen, and B. de Wit, Nucl. Phys. B154, 261 (1979), Note, that there is an obvious missprint in definition of the $\operatorname{spin} \mathcal{P}_{22 \rho \sigma ; \tau \delta}^{\frac{3}{2}}(p)$ projector in (A.1): the last term $(-1 / 15) \theta_{\mu \nu} \theta_{\rho \sigma}$ should read $(-2 / 15) \theta_{\mu \nu} \theta_{\rho \sigma}$.

[21] J. C. David, C. Fayard, G. H. Lamot, and B. Saghai, Phys. Rev. C53, 2613 (1996).

[22] M. Zetenyi and G. Wolf, Heavy Ion Phys. 17, 27 (2003), nucl-th/0202047.

[23] S. Weinberg and E. Witten, Phys. Lett. B96, 59 (1980).

[24] K. H. Glander, et al. (SAPHIR Collaboration), Eur. Phys. J. A19, 251 (2004).

[25] The CLAS collaboration, J. W. C. McNabb et al., Phys. Rev. C69, 042201 (2004)

[26] R. D. Baker et al., Nucl. Phys. B141, 29 (1978).

[27] D. H. Saxon et al., Nucl. Phys. B162, 522 (1980).

[28] T. M. Knasel et al., Phys. Rev. D11, 1 (1975).

[29] R. G. T. Zegers et al. (LEPS Collaboration), Phys. Rev. Lett. 91, 092001 (2003).

[30] M. Sumihama et al. (LEPS Collaboration), Phys. Rev. C 73, 035214 (2006).

[31] H. Kohri et al. (LEPS Collaboration), Phys. Rev. Lett. 97, 082003 (2006).

[32] R. Bradford et al. (CLAS Collaboration), Phys. Rev. C 73, 035202 (2006).

[33] B. Dey et al. (CLAS Collaboration), Phys. Rev. C 82, 025202 (2010).

[34] Crystal Ball at MAMI, E. F. McNicoll et al., Phys. Rev. C82, 035208 (2010).

[35] CLAS Collaboration, M. Dugger et al., Phys.Rev.Lett. 89, 222002 (2002).

[36] CBELSA/TAPS Collaboration, V. Crede et al., Phys.Rev. C80, 055202 (2009).

[37] CB-ELSA Collaboration, O. Bartholomy et al., Eur.Phys.J. A33, 133 (2007).
[38] The GRAAL collaboration, O. Bartalini et al., Eur.Phys.J. A33, 169 (2007).

[39] R. M. Davidson and R. Workman, Phys. Rev. C63, 025210 (2001).

[40] R. Arndt, W. Briscoe, I. Strakovsky, and R. Workman, Phys.Rev. C74, 045205 (2006), nucl-th/0605082.

[41] Particle Data Group, J. Beringer et al., Phys.Rev. D86, 010001 (2012).

[42] D. M. Manley, R. A. Arndt, Y. Goradia, and V. L. Teplitz, Phys. Rev. D30, 904 (1984).

[43] A. Lleres et al. (GRAAL-ESRF Collaboration), Eur. Phys. J. A31, 79 (2007).

[44] B. Carnahan, Ph.D. thesis, The Catholic University of America, 2003.

[45] R. Castelijns et al. (CBELSA/TAPS Collaboration), Eur. Phys. J. A35, 39 (2008).

[46] CB-ELSA Collaboration, V. Crede et al., Phys.Rev.Lett. 94, 012004 (2005), hep-ex/0311045. ev. D84, 074508 (2011).

[47] S. Dürr et al., Science 322, 1224 (2008).

[48] R. G. Edwards, J. J. Dudek, D. G. Richards, and S. J. Wallace, Phys.R

[49] H. Sanchis-Alepuz, G. Eichmann, S. VillalbaChavez, and R. Alkofer, Phys.Rev. D84, 096003 (2011).

[50] R. Koniuk and N. Isgur, Phys. Rev. D 21, 1868 (1980).

[51] S. Capstick and W. Roberts, Phys.Rev. D58, 074011 (1998).

[52] R. Cutkosky, C. Forsyth, R. Hendrick, and R. Kelly, Phys.Rev. D20, 2839 (1979).

[53] G. Hoehler, PiN Newslett. 9, 1 (1993).

[54] A. Anisovich et al., Eur.Phys.J. A48, 15 (2012).

[55] R. A. Arndt, J. M. Ford, and L. D. Roper, Phys.Rev. D32, 1085 (1985).

[56] R. Cutkosky and S. Wang, Phys.Rev. D42, 235 (1990).

[57] M. Döring, C. Hanhart, F. Huang, S. Krewald, and U.-G. Meißner, Nucl.Phys. A829, 170 (2009).

[58] N. Suzuki et al., Phys.Rev.Lett. 104, 042302 (2010).

[59] Crystal Ball Collaboration, K. Craig et al., Phys.Rev.Lett. 91, 102301 (2003).

[60] Crystal Ball Collaboration, S. Prakhov et al., Phys.Rev. C69, 045202 (2004).

[61] T. Skorodko et al., Eur.Phys.J. A35, 317 (2008). 